\title{
Optimization Research in Colour Semantics of Smart Phone Interface Icons for the Elderly
}

\author{
Zhi-Zheng ZHANG, Ya-Jun LI
}

School of Design Art\&Media, Nanjing University of Science and Technology, 210094, China

450724699@qq.com

${ }^{*}$ Corresponding author

Keywords: Elderly,Colour Semantics,Interface Icons.

\begin{abstract}
With today's rapidly aging society, there is concern about optimizing designs for elderly consumers. In this research, we perform Spearman's rank correlation coefficient analysis to study the colour satisfaction rate for smartphone interface icons and to determine whether there is a correlation with the colour semantics. In this experiment, we used questionnaires, the perceptual engineering image scale method and mathematical analysis. In three phases, we verified the icon colour satisfaction rate of elderly users and its correlation with colour semantics. The results indicate the relevance of and positive correlation between elderly users' satisfaction rates with the smartphone icon colours and colour semantics. Using this research methodology, the results can provide guidance on optimization research for the elderly man-machine interface.
\end{abstract}

\section{Introduction}

As society advances and the world's population ages, designs for products for the elderly have begun to attract increasing attention. Improvements in living quality combined with the unique needs of the elderly have expanded the expectations of elderly smartphone users. The man-machine interface between the elderly and the products they consume influences the accuracy and ready availability of the product information at hand. Colour, a main element in the man-machine interface, is an important style factor, and the use of colour concepts and innovation have a significant influence on satisfaction rates. Optimization research in smart phone applications for the elderly with respect to colour semantics plays an important role in improving the user-friendliness and acceptance of smartphone interfaces.

In this study, we used questionnaires to obtain data regarding the satisfaction levels regarding icon colours of elderly smartphone users. Based on a perceptual engineering scale, we extracted the colour semantics vocabulary of elderly users. To evaluate the user satisfaction with respect to icon colours and their colour semantics vocabulary, we analysed the relationship between the two using Spearman's rank correlation coefficient and, based on our experiment results, we propose an approach for optimizing smartphone icon designs for the elderly.

Colour Semantics. Colour semantics is a concept that was first proposed for the introduction of colour connotations in product semantics to enhance product quality. Since individuals have a relatively consistent recognition of colours, the symbolic and information dissemination functions of colours can be optimized.[1] The significance of colour has long been established in social practice, and has thus led to the formation of stable colour semantics. To optimize designs of man-machine interfaces for the elderly, colour semantics that are familiar to elderly users should be chosen. 
Variations in the Reorganization of Colour Semantics. There are variations and identifiable groupings in people's perception of colour semantics.

Examples of variations in individual perception of colour semantics include differences in social, historical and life experience factors. Each individual has different comprehensions of colour,[2] and for one individual, advancing age will bring changes in colour preferences along with psychological changes that will generate different colour perceptions.

In the identification of any two individuals' perceptions of colour semantics, we find that their perceptions and associations of colours cannot possibly be identical. However, under certain colour contexts, i.e., such as the same life circumstances, cultural background or living environment, they will have similar features.

In this research, we considered the factors influencing the perception of colour semantics, and selected a number of elderly and young people as test subjects, based on some screening conditions: (1) the elderly people were aged between 65 and 75; (2) the young people were aged between 18 and 30; (3) all test subjects had relatively high economic and living standards; and (4) all test subjects had owned smartphones for more than three months and had experience using them.

Finally, we selected 40 elderly and 40 young test subjects, and compared the experimental data of each group to identify variations in their icon colour satisfaction rates and in the colour semantics of elderly smartphone users, as well as the correlation between the two.

\section{Methods}

Binary variable correlation analysis refers to the analysis of the degree of correlation between two or more variables by calculating the correlation coefficient between them. There are three common types of binary variable correlation coefficients, including the Pearson simple correlation coefficient, Spearman's rank correlation coefficient and the Kendall rank correlation coefficient.[3]

In this study, we applied Spearman's rank correlation coefficient analysis in order to correlate the icon colour satisfaction rate and the colour semantics of elderly smartphone users. Using Spearman's rank correlation coefficient, we estimated the correlation between these variants, labelled $\mathrm{X}$ and $\mathrm{Y}$, respectively, using the monotonic function shown in Formula (1):

$$
\rho=\frac{\sum_{i-1}^{N}\left(X_{i}-\bar{X}\right)\left(Y_{i}-\bar{Y}\right)}{\sqrt{\sum_{i=1}^{N}\left(X_{i}-\bar{X}\right)^{2} \sum_{i=1}^{N}\left(Y_{i}-\bar{Y}\right)^{2}}}
$$

The experiment process comprised three phases, as shown in Fig. 1.

Phase 1: We established the icon colour samples for the experiment and developed a satisfaction grading system for the icon colour satisfaction rate of the elderly smartphone users for hue-saturation-luminance (HSV) icon colour samples, which were then transformed into red-green-blue (RGB) colour samples.

Phase 2: We established samples of the icon colour semantics.

Phase 3: The elderly test subjects graded the corresponding semantic samples of the smartphone icon colours, and we conducted a Spearman's rank correlation coefficient analysis to determine the correlation between the icon colour satisfaction rate and the colour semantics of elderly smartphone users. 


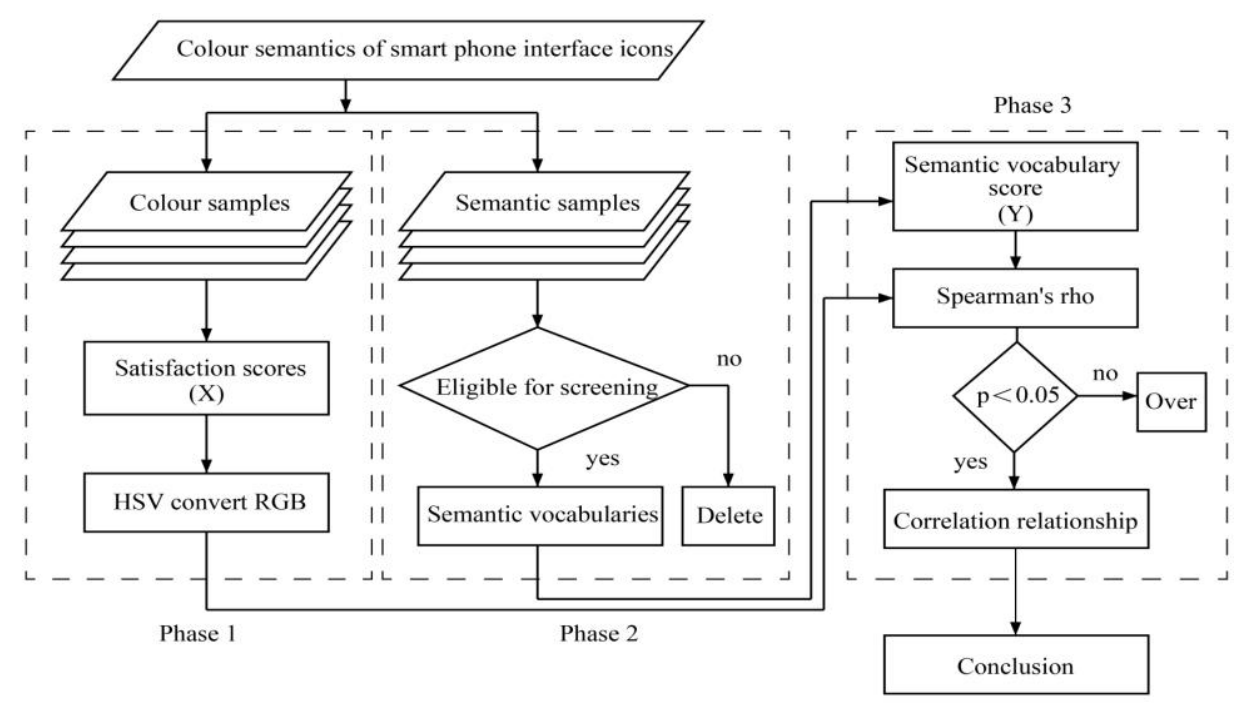

Fig. 1 Optimization research on colour semantics of smartphone interface icons for the elderly

Phase I: Establishment of Icon Colour Samples. Based on the Munsell colour order system, we quantitatively established the initial HSV icon colour bank (including colour coding and the property values of various colours), based on three colour property values in the icon colour bank, for which matching colours were harmonized.

Quantitative Methods for HSV Icon Colours. In the Munsell colour order system, colours are organized according to three basic properties: hue $(\mathrm{H})$, saturation $(\mathrm{S})$ and luminance $(\mathrm{V})$, and these properties each have their own value range: $\mathrm{H} 0-360$, S $0-100$, and V $0-100$. We opted to use the HSV colour model for two reasons. First, the values for V (luminance) and colour information (hue and saturation) are decoupled. Second, the HSV colour model describes perceptual colour relationships more accurately than does the RGB colour model.[4] Since these three properties feature three dimensions, a mutually vertical coordinate system for the three coordinates of $\mathrm{x}, \mathrm{y}$ and $\mathrm{z}$ can be established, in which any given point can express a unique and corresponding colour.[5] The function of any colour can be uniquely determined by the three properties shown in Formula (2).

$$
C=f(x, y, z)
$$

This method uses a colorimeter and digital photography to obtain XYZ colour space values, which are then mathematically transformed into HSV cylindrical-coordinates.[6] In the formula, $x$, $\mathrm{y}$ and $\mathrm{z}$ respectively represent three-dimensional values where $\mathrm{C}$ is colour, $\mathrm{x}$ is hue, $\mathrm{y}$ is saturation and $\mathrm{z}$ is luminance. In our study, the value ranges of these variants were: $0 \leq \mathrm{x} \leq 360,0 \leq \mathrm{y} \leq 100$ and $0 \leq \mathrm{z} \leq 100$.

Construction of Colour Samples. Based on the above colour value ranges and the formulas for quantifying colour, in this study we established sectional value ranges for the three colour dimensions and obtained a certain number of colour samples using combinations. Specifically, in light of what is known about the visual characteristics of elderly people, we adopted the following research-based sectional value ranges: Hue $(\mathrm{H})$ was divided into seven sections, with the values $\mathrm{H}=$ 0, 60, 120, 180, 240, 300 and 360. Saturation (S) was divided into five sections, with $S=20,40,60$, 80 and 100. Luminance (V) was divided into four sections, with $\mathrm{V}=20,40,60$ and 80 . Thus, we generated a total of $140(7 * 5 * 4)$ icon colour samples, and the samples and their property values are shown in Table 1. 
Table 1. Coding of icon colour samples and their property values

\begin{tabular}{cccccccc}
\hline No. & $\mathbf{H , S}, \mathbf{V}$ & No. & $\mathbf{H , S}, \mathbf{V}$ & No. & $\mathbf{H , S , V}$ & No. & $\mathbf{H , S , V}$ \\
\hline 1 & $0,20,20$ & 36 & $60,80,80$ & 71 & $120,80,80$ & 106 & $240,40,40$ \\
2 & $0,20,40$ & 37 & $60,100,20$ & 72 & $120,100,20$ & 107 & $240,40,60$ \\
3 & $0,20,60$ & 38 & $60,100,40$ & 73 & $120,100,40$ & 108 & $240,40,80$ \\
4 & $0,20,80$ & 39 & $60,100,60$ & 74 & $120,100,60$ & 109 & $240,60,20$ \\
5 & $0,40,20$ & 40 & $60,100,80$ & 75 & $120,100,80$ & 110 & $240,60,40$ \\
6 & $0,40,40$ & 41 & $120,20,20$ & 76 & $180,20,20$ & 111 & $240,60,60$ \\
$\vdots$ & $\vdots$ & $\vdots$ & $\vdots$ & $\vdots$ & $\vdots$ & $\vdots$ & $\vdots$ \\
35 & $60,80,60$ & 70 & $120,80,60$ & 105 & $240,40,20$ & 140 & $360,100,80$ \\
\hline
\end{tabular}

Affective user satisfaction is widely considered to be one of the most important factors with respect to consumer product use.[7] In the experiment, 40 elderly users and 40 young users conducted a semantics evaluation from "Dissatisfied" to "Satisfied" for the colour samples of the 140 icon colours, in a numerical evaluation range from 1-7, where " 1 " indicates highly dissatisfied, "2" dissatisfied, "3" rather dissatisfied, "4" neutral , "5" rather satisfied, "6" satisfied and "7" highly satisfied. We then statistically calculated the subjective satisfaction grades of the evaluation values, as shown in Table 2.

Based on the results, we can see that the satisfaction levels of the elderly test subjects differed from those of the young test subjects. For different hues, the evaluation grades of these two groups also differed. The young test subjects had higher satisfaction rates for icon colours with high saturation, while the elderly test subjects had higher satisfaction rates on colours with high luminance. Since there were certain differences between the subjective icon colour satisfaction rates for the elderly and young test subjects, we found that our study of colour semantic vocabularies was not applicable to the elderly group, and it became necessary to study the relationship between the satisfaction rate and colour semantics with respect to the colours preferred by the elderly users.

Table 2. Relevant values of the satisfaction rate evaluation of the 140 icon color samples

\begin{tabular}{ccccccccccccc}
\hline \multicolumn{1}{c}{ Elderly users } & \multicolumn{1}{c}{ Young users } \\
\hline No & H,S,V & Sc & No & H,S,V & Sco & No & H,S,V & Sco & N & H,S,V & Sco \\
1 & $0,20,2$ & $\mathbf{0 . 2}$ & 10 & $60,80,8$ & $\mathbf{5 . 6}$ & 1 & $0,20,20$ & $\mathbf{1 . 7}$ & 10 & $60,80,8$ & $\mathbf{4 . 5}$ \\
2 & $0,20,4$ & $\mathbf{0 . 3}$ & 10 & 60,100, & $\mathbf{5 . 3}$ & 2 & $0,20,40$ & $\mathbf{1 . 8}$ & 10 & 60,100, & $\mathbf{5 . 5}$ \\
3 & $0,20,6$ & $\mathbf{0 . 7}$ & 10 & 60,100, & $\mathbf{5 . 4}$ & 3 & $0,20,60$ & $\mathbf{1 . 1}$ & 10 & 60,100, & $\mathbf{5 . 6}$ \\
4 & $0,20,8$ & $\mathbf{2 . 8}$ & 10 & 60,100, & $\mathbf{4 . 9}$ & 4 & $0,20,80$ & $\mathbf{2 . 0}$ & 10 & 60,100, & $\mathbf{5 . 7}$ \\
5 & $0,40,2$ & $\mathbf{1 . 5}$ & 11 & 60,100, & $\mathbf{3 . 8}$ & 5 & $0,40,20$ & $\mathbf{2 . 6}$ & 11 & 60,100, & $\mathbf{5 . 7}$ \\
6 & $0,40,4$ & $\mathbf{3 . 2}$ & 11 & 120,20, & $\mathbf{5 . 9}$ & 6 & $0,40,40$ & $\mathbf{2 . 6}$ & 11 & 120,20, & $\mathbf{5 . 1}$ \\
$\vdots$ & $\vdots$ & $\vdots$ & $\vdots$ & $\vdots$ & $\vdots$ & $\vdots$ & $\vdots$ & $\vdots$ & $\vdots$ & $\vdots$ & $\vdots$ \\
10 & 240,4 & $\mathbf{5 . 0}$ & 14 & 360,10 & $\mathbf{6 . 0}$ & 10 & 240,40, & $\mathbf{5 . 3}$ & 14 & 360,100 & $\mathbf{6 . 5}$ \\
\hline
\end{tabular}

Based on the evaluation value ranking of the satisfaction rates, a total of ten colour samples received satisfactory evaluations by the elderly users, and we used these values in Phase III. In order show these samples on smartphones in Phase III of the experiment, we used Formulas (3) and (4) to transform the ten groups of HSV colours into RGB colours, and then configured these ten 
groups of smartphone icon colour schemes for smartphones. The R, G and B values of the colours of the various schemes are shown in Table 3.

$$
\begin{aligned}
& h_{i}=\left|\frac{H}{60}\right| \bmod 6 \\
& f=\frac{H}{60}-h_{i} \\
& p=v \times(1-s) \\
& q=v \times(1-f \times s) \\
& t=v \times(1-(1-f) \times s) \\
& (R, G, B)= \begin{cases}(v, t, p), & \text { if } h_{i}=0 \\
(q, v, p), & \text { if } h_{i}=1 \\
(p, v, t), & \text { if } h_{i}=2 \\
(p, q, v), & \text { if } h_{i}=3 \\
(t, p, v), & \text { if } h_{i}=4 \\
(v, p, q), & \text { if } h_{i}=5\end{cases}
\end{aligned}
$$

These ten groups of icon colour sample schemes served as our experimental test materials in our verification of whether there is a correlation between the colour satisfaction rates of elderly smartphone users and the variants of their colour semantics.

Table 3. The RGB values for the icon colour schemes of 10 groups of elderly users

\begin{tabular}{cccccccccc}
\hline No & $\mathbf{R}, \mathbf{G}, \mathbf{B}$ & No. & $\mathbf{R , G}, \mathbf{B}$ & No. & $\mathbf{R , G}, \mathbf{B}$ & No. & $\mathbf{R , G , B}$ & No. & $\mathbf{R , G , B}$ \\
$\cdot$ & & & & & & & & \\
$\mathrm{C}_{1}$ & $204,0,0$ & $\mathrm{C}_{3}$ & $20,102,20$ & $\mathrm{C}_{5}$ & $0,51,0$ & $\mathrm{C}_{7}$ & $102,22,102$ & $\mathrm{C}_{9}$ & $153,0,0$ \\
$\mathrm{C}_{2}$ & $204,204,41$ & $\mathrm{C}_{4}$ & $41,102,41$ & $\mathrm{C}_{6}$ & $31,31,153$ & $\mathrm{C}_{8}$ & $153,61,153$ & $\mathrm{C}_{10}$ & $204,43,43$ \\
\hline
\end{tabular}

Phase II: Establishment of the Samples of Icon Colour Semantics. Considering that the perception of icon colour semantics may vary from person to person, to establish reasonable and general semantics samples, we conducted survey screening by questionnaire regarding appropriate semantic vocabularies for the icon colours. These questionnaires were given to the 40 elderly and 40 young test subjects. T1 represents the number of choices by the elderly test subjects, and T2 the number of choices by the young test subjects, the results of which are shown in Table 4.

Based on the data in Table 4, for the same icon colour semantic vocabularies, the number of choices differed for these two groups of test subjects, which indicates a big variation between their semantic vocabularies. Of the 20 colour vocabularies, the icon colour vocabulary sizes of the elderly test subjects were smaller than those of the young test subjects. This finding indicates that some limitations are associated with age in the breadth of the semantic vocabulary of colours. Hence, it is necessary to select colour semantics within the recognition range of elderly smartphone users as research variants. 
Table 4. Statistical data of the test subjects on the semantic vocabularies of icon colours

\begin{tabular}{cccccccc}
\hline No. & Vocabulary & $\mathbf{T}_{\mathbf{1}}$ & $\mathbf{T}_{\mathbf{2}}$ & No. & Vocabula & $\mathbf{T}_{\mathbf{1}}$ & $\mathbf{T}_{\mathbf{2}}$ \\
\hline $\mathrm{V}_{1}$ & Calm & $\mathbf{2 8}$ & $\mathbf{2 2}$ & $\mathrm{V}_{11}$ & Amiable & $\mathbf{2 6}$ & $\mathbf{3 0}$ \\
$\mathrm{V}_{2}$ & Spiritual & $\mathbf{9}$ & $\mathbf{2 5}$ & $\mathrm{V}_{12}$ & Clear- & $\mathbf{2 1}$ & $\mathbf{2 7}$ \\
$\mathrm{V}_{3}$ & Relaxing & $\mathbf{2 3}$ & $\mathbf{3 5}$ & $\mathrm{V}_{13}$ & Worry- & $\mathbf{2 5}$ & $\mathbf{2 8}$ \\
$\mathrm{V}_{4}$ & Decisive & $\mathbf{2 5}$ & $\mathbf{3 0}$ & $\mathrm{V}_{14}$ & Devoted & 11 & $\mathbf{1 6}$ \\
$\mathrm{V}_{5}$ & Enthusiastic & $\mathbf{3 1}$ & $\mathbf{2 7}$ & $\mathrm{V}_{15}$ & Confident & $\mathbf{2 1}$ & $\mathbf{2 3}$ \\
$\mathrm{V}_{6}$ & Unperturbed & $\mathbf{1 7}$ & $\mathbf{2 9}$ & $\mathrm{V}_{16}$ & Enlighteni & $\mathbf{2 7}$ & $\mathbf{2 3}$ \\
$\mathrm{V}_{7}$ & Dedicated & $\mathbf{3 2}$ & $\mathbf{2 8}$ & $\mathrm{V}_{17}$ & Elemental & $\mathbf{1 5}$ & $\mathbf{2 0}$ \\
$\mathrm{V}_{8}$ & Pure & $\mathbf{3 8}$ & $\mathbf{3 5}$ & $\mathrm{V}_{18}$ & Active & $\mathbf{3 3}$ & $\mathbf{3 1}$ \\
$\mathrm{V}_{9}$ & Cozy & $\mathbf{2 7}$ & $\mathbf{2 3}$ & $\mathrm{V}_{19}$ & Friendly & $\mathbf{2 6}$ & $\mathbf{3 6}$ \\
$\mathrm{V}_{10}$ & Peaceful & $\mathbf{7}$ & $\mathbf{2 6}$ & $\mathrm{V}_{20}$ & Exciting & $\mathbf{3 0}$ & $\mathbf{3 4}$ \\
\hline
\end{tabular}

Based on the principle of a majority being "more than half," we selected the final samples of the semantic vocabularies of the icon colours, for those in which the number of choices were more than half of the total number. We eliminated vocabularies whose number of choices was lower than 20 a total of 5 vocabularies - as shown in grey shadows in Table 4. Following the elimination and recoding process, a total of 15 semantic samples of icon colours were derived, as shown in Table 5 .

Table 5. Samples of the semantics of icon colours for the elderly people

\begin{tabular}{cccccc}
\hline No. & Vocabulary & No. & Vocabulary & No. & Vocabulary \\
\hline $\mathrm{V}_{1}$ & Calm & $\mathrm{V}_{6}$ & Pure & $\mathrm{V}_{11}$ & Confident \\
$\mathrm{V}_{2}$ & Relaxing & $\mathrm{V}_{7}$ & Cozy & $\mathrm{V}_{12}$ & Enlightening \\
$\mathrm{V}_{3}$ & Decisive & $\mathrm{V}_{8}$ & Amiable & $\mathrm{V}_{13}$ & Active \\
$\mathrm{V}_{4}$ & Enthusiastic & $\mathrm{V}_{9}$ & Clear-minded & $\mathrm{V}_{14}$ & Friendly \\
$\mathrm{V}_{5}$ & Dedicated & $\mathrm{V}_{10}$ & Worry-free & $\mathrm{V}_{15}$ & Exciting \\
\hline
\end{tabular}

\section{Phase III: Relationship between the Satisfaction Rate and the Colour Semantics of Colour Icons.}

Grading of the Colour Semantics. We used a total of ten icon colour schemes and asked the 40 elderly test subjects to evaluate the icon colour semantics samples in the smartphone interfaces. This questionnaire was based on a seven-point binary Likert scale.[9] In combination with the scores for satisfaction with icon colours (S), we derived scores for the satisfaction rate and semantics vocabulary of the ten icon colour schemes, as shown in Table 6. (Due to the space limitations, only partial experiment data are shown.) 
Table 6. The scores of the satisfaction rate and semantics vocabulary of the $\mathrm{C}_{1}$ groups of icon colours

\begin{tabular}{ccccccccccccccccc}
\hline No. & $\mathbf{V}$ & $\mathbf{V}$ & $\mathbf{V}$ & $\mathbf{V}_{\mathbf{4}}$ & $\mathbf{V}$ & $\mathbf{V}_{\mathbf{6}}$ & $\mathbf{V}$ & $\mathbf{V}$ & $\mathbf{V}$ & $\mathbf{V}$ & $\mathbf{V}$ & $\mathbf{V}$ & $\mathbf{V}$ & $\mathbf{V}$ & $\mathbf{V}$ & $\mathbf{S}$ \\
\hline $\mathbf{1}$ & 1 & 1 & 4 & 7 & 6 & 5 & 5 & 7 & 4 & 3 & 4 & 1 & 7 & 3 & 5 & 6 \\
$\mathbf{2}$ & 1 & 1 & 5 & 6 & 5 & 4 & 1 & 4 & 4 & 2 & 4 & 2 & 5 & 2 & 5 & 5 \\
$\mathbf{3}$ & 1 & 1 & 3 & 7 & 6 & 4 & 3 & 6 & 3 & 2 & 5 & 4 & 5 & 4 & 6 & 3 \\
$\mathbf{4}$ & 1 & 1 & 4 & 7 & 6 & 1 & 3 & 5 & 4 & 3 & 5 & 4 & 7 & 4 & 3 & 5 \\
$\mathbf{5}$ & 1 & 1 & 7 & 7 & 7 & 2 & 2 & 5 & 4 & 4 & 4 & 3 & 5 & 2 & 5 & 7 \\
$\mathbf{6}$ & 1 & 1 & 5 & 5 & 6 & 1 & 3 & 5 & 4 & 3 & 7 & 1 & 6 & 3 & 5 & 6 \\
$\vdots$ & $\vdots$ & $\vdots$ & $\vdots$ & $\vdots$ & $\vdots$ & $\vdots$ & $\vdots$ & $\vdots$ & $\vdots$ & $\vdots$ & $\vdots$ & $\vdots$ & $\vdots$ & $\vdots$ & $\vdots$ & $\vdots$ \\
$\mathbf{4 0}$ & 1 & 5 & 6 & 6 & 6 & 4 & 3 & 5 & 3 & 4 & 4 & 3 & 4 & 4 & 7 & 5 \\
\hline
\end{tabular}

Spearman Rank Correlation Coefficient Analysis. When the statistical significance of the correlation coefficient test results is $\mathrm{p}<0.05$, this indicates that there is a significant correlation between the semantic vocabularies for icon colours and the colour satisfaction rates. When $\mathrm{p}<0.01$, this indicates a correlation between the semantic vocabulary of this icon colour and the satisfaction rates. When $\mathrm{p}>0.05$, this means there is no significant correlation between the semantic vocabularies of the icon colours and the colour satisfaction rates.[8] We analysed the results for the ten icon colour schemes using Spearman's rank correlation coefficient and the correlation factors between the 15 semantic vocabularies for icon colours and the colour satisfaction rates. The analysis results are shown in Table 7. (Due to the space limitations, only partial experiment data are shown.)

Table 7. Relevant coefficient data of the semantic vocabularies of $\mathrm{C}_{1}$ icon and the satisfaction rates

\begin{tabular}{ccccc}
\hline & & \multicolumn{3}{c}{$\mathbf{S}$} \\
\cline { 3 - 5 } & & Correlation Coefficient & Sig. (p) & $\mathbf{N}$ \\
\hline & $\mathrm{V}_{1}$ & $\cdot$ & $\cdot$ & 40 \\
& $\mathrm{~V}_{2}$ & $\cdot$ & $\cdot$ & 40 \\
& $\mathrm{~V}_{3}$ & -0.420 & 0.407 & 40 \\
& $\mathrm{~V}_{4}$ & 0.632 & 0.178 & 40 \\
& $\mathrm{~V}_{5}$ & $0.894^{*}$ & 0.016 & 40 \\
& $\mathrm{~V}_{6}$ & -0.525 & 0.285 & 40 \\
& $\mathrm{~V}_{7}$ & 0.210 & 0.690 & 40 \\
rho & $\mathrm{V}_{8}$ & $0.853^{*}$ & 0.031 & 40 \\
& $\mathrm{~V}_{9}$ & 0.250 & 0.633 & 40 \\
& $\mathrm{~V}_{10}$ & 0.335 & 0.516 & 40 \\
& $\mathrm{~V}_{11}$ & -0.367 & 0.474 & 40 \\
& $\mathrm{~V}_{12}$ & -0.210 & 0.690 & 40 \\
& $\mathrm{~V}_{13}$ & 0.000 & 1.000 & 40 \\
& $\mathrm{~V}_{14}$ & -0.107 & 0.841 & 40 \\
& $\mathrm{~V}_{15}$ & -0.440 & 0.383 & 40 \\
\hline
\end{tabular}

In our two-element correlation analysis, in the $\mathrm{C} 1$ icon colour scheme the correlations between the semantic vocabularies coded V2, V5 and V6 were significant; and in the C2 icon colour scheme the correlation between the semantic vocabulary coded V15 was significant. In the C3 icon colour scheme the correlations between the semantic vocabularies coded V2, V5 and V6 and the satisfaction rate $(\mathrm{S})$ were significant; and in the $\mathrm{C} 4$ icon colour scheme the correlations between the semantic vocabularies coded V5 and V6 and the satisfaction rate (S) were significant, and the 
correlation between the semantic vocabulary of V7 and the satisfaction rate was very significant. In the C5 icon colour scheme the correlations between the semantic vocabularies coded V7, V12 and V14 and the satisfaction rate (S) were significant; and in the C6 icon colour scheme the correlations between the semantic vocabularies coded V1 and V6 and the satisfaction rate (S) were significant. In the $\mathrm{C} 7$ icon colour scheme the correlations between the semantic vocabularies coded V6, V7 and V8 and the satisfaction rate (S) were significant; and in the C8 icon colour scheme the correlation between the semantic vocabularies coded V2 and the satisfaction rate (S) was high. In the C9 icon colour scheme, there was no correlation between the semantic vocabularies and the satisfaction rate (S); and in the C10 icon colour scheme the correlations between the semantic vocabularies coded $\mathrm{V} 4$ and V5 and the satisfaction rate (S) were significant.

\section{Results and Discussion}

We conducted a two-element correlation analysis on the satisfaction rates with icon colours of elderly smartphone users and their colour semantic vocabularies, which were transformed into 15 colour semantic vocabularies. Based on a significance level less than 0.05 , we determined whether there was a correlation between colour semantic vocabulary and colour satisfaction rate. Our statistical results revealed that among the ten groups of Spearman's rank correlation coefficient data, nine had significant correlations, and all correlations were positive. Of these, the correlation coefficients of V5 and V6 with the ten icon colour schemes were highest, and of V3, V9, V10, V11, and V13, not a single icon colour scheme had any correlation. The higher the recognition of the colour semantic vocabularies, the higher the users' satisfaction rates for that colour.

The correlation coefficients of the different semantic vocabularies vary for the colour of each icon. Using the icon colour scheme $\mathrm{C} 1$ as an example, the semantic vocabularies that correlated with the satisfaction rate were V5 and V8, for which the correlation coefficient of V5 was 0.894, with a significance of 0.016 ; and the correlation coefficient of V8 was 0.853 , with a significance 0.031 . Since $0.016<0.031$, this indicates that the correlation of V5 with the icon colour of $\mathrm{C} 1$, was greater than that of $\mathrm{V} 8$.

An understanding of the semantics of different colours for elderly users is essential to better develop product interfaces to convey product information and to ensure more efficient man-machine interactions. By combining elderly smartphone users' satisfaction rates regarding icon colours with their colour semantics and quantifying and analysing their perceptual experiences, research results can be applied to optimize the design of products for the elderly. For example, research findings can be used to develop effective colour configurations for industrial products and man-machine interfaces for elderly consumers. Colour semantics can be used in scales of consumer satisfaction with respect to meeting the psychological and physiological needs of elderly consumers and in designing suitable products.

\section{Conclusions}

In this research, we focused on developing a method to determine the correlation between colour semantics and satisfaction rates regarding icon colours. We quantified the emotional vocabularies and performed mathematical analysis to more accurately assess elderly peoples' perceptions of the semantics of colour. In doing so, we sought to arouse the memories of elderly smartphone users and to better understand their psychology, which are the main goals in the process of optimizing designs for elderly people's interface with machines.

In our study, elderly users had relatively higher recognition of the colour semantics of the V5, V6, V8 and V2 icons, and these colour vocabularies had significant impacts on their satisfaction rates regarding the icon colours. In optimized designs of the man-machine interface for the elderly, we found that it is possible use more corresponding RGB colours, including C1 $(204,0,0)$, C4 $(41,102,41)$, C6 $(31,31,153), C 7(102,22,102), C 8(153,61,153)$ and C10 $(204,43,43)$ to improve the acceptance of man-machine interfaces by elderly smartphone users and to enhance their experiences 
and operation efficiencies. When designing the colour of man-machine interfaces for elderly smartphone users, research to optimize colour semantics is of particular importance.

\section{References}

[1] G.S. Choi, R. Oehlmann, H. Dalke,D. Cottington. LNAI 4693, pp. 926-933 (2007)

[2] D. Man , D. Wei , Y. Chih-Chieh. Journal of Mechanical Science and Technology 27, pp. 2079-2084 (2013)

[3] P. Chen , J. Zhang, L. Zhang, Y. Sun. International Journal of Environmental Research and Public Health 11(10), pp. 9964-9980 (2014)

[4] C.M. Tsai, Z.M. Yeh, IEEE Transactions on Consumer Electronics 54(2), pp. 213-219 (2008)

[5] X. Jing, T. Yue, L. Zheng, Proceedings of International Conference on Computer Science and Information Technology 255, pp. 663-669(2014)

[6] M.R. Olson, E. Graham, S. Hamad, P. Uchupalanun, N. Ramanathan, J.J. Schauer, The Science of The Total Environment 548, pp. 252-259 (2016)

[7] J. Park, S.H. Han, International Journal of Industrial Ergonomics 34, pp. 31-47 (2014)

[8] W.K. Härdle, L. Simar, Applied Multivariate Statistical Analysis (2015)

[9] M. Yoda, A. Yoda, Y Shiota, JSME International Journal Series C 49(4), pp. 1100-1106 (2006) 